\title{
Spinal Dural Arteriovenous Fistula-Induced Myelopathy: Rare and Commonly Missed but Treatable Nevertheless
}

\author{
Manish Sharma ${ }^{1} \quad$ Ankit Mathur ${ }^{2}$ \\ ${ }^{1}$ Department of Neurosurgery, Command Hospital (EC), Kolkata, \\ West Bengal, India \\ 2Department of Interventional Radiology, Army Hospital (R\&R), \\ New Delhi, India \\ Indian J Neurosurg 2019;8:108-112
}

\begin{abstract}
Address for correspondence Ankit Mathur, DM Neuroradiology, Department of Radiology, Army Hospital (R\&R) Delhi Cantt. 110010 New Delhi, India (e-mail: ankitmathur74@yahoo.com).
\end{abstract}

\begin{abstract}
Objectives To highlight spinal dural arteriovenous fistula (dAVF) as a treatable cause of myelopathy that is often misdiagnosed. Clinical progression, hallmark radiological findings, and postoperative course of recovery are studied.

Patients and Methods Three patients with spinal dAVF were included in the study. Retrospective evaluation of the progression of disease and treatment received was done. Two of them were managed by surgical ligation of fistula, and the third patient was under observation because of lack of localizing symptoms.

Results Two of the three patients had previously been diagnosed with multiple sclerosis, and one of them had received steroids. The third case was easily diagnosed at an early stage as the center had become adequately sensitized about the radiologic findings. After surgical excision of the fistula, both operated cases recovered steadily but significantly, regained significant neurologic functions despite being operated at an advanced stage.

Keywords Conclusion Spinal dAVF is a rare entity. They have the potential to cause severe morbidity in the form of myelopathic changes in the cord and paraparesis in advanced stages. They can be misdiagnosed even after a magnetic resonance imaging evaluation as the appearance of dilated perimedullary veins and cord changes are not easy to interpret and require specific understanding of the radiologic changes that may occur. However, once diagnosis has been established, an early interruption of the fistula leads to a good clinical recovery.
\end{abstract}

\section{Introduction}

Spinal dural arteriovenous fistula (dAVF) is the most common type of spinal arteriovenous (AV) malformation but still is a rare entity. Its clinical presentation is vague and nonspecific. It frequently goes undiagnosed and is often misdiagnosed. This leads to late diagnosis and consequently delayed treatment and incomplete recovery. Initial suspicion of this disease invariably is made when dilated venous architecture is incidentally picked up on a magnetic resonance imaging (MRI) while investigating a patient for some other clinical diagnosis. The diagnosis can be missed even after doing an MRI, unless those familiar with this entity scrutinize the MRI for more obvious cord changes that have already developed. We discuss our experience including diagnostic challenges, therapeutics, and outcome of three such cases over 6 months and highlight the fact that an early intervention leads to rapid and good recovery.

\section{Anatomy}

The blood supply to the spine and the spinal cord is derived from various segmental arteries (vertebral arteries, deep and ascending cervical arteries, intercostal arteries, lumbar and iliolumbar arteries). At each segment, there is a radicular received

September 11, 2018

accepted

November 13, 2018

published online

August 13, 2019
DOI https://doi.org/

$10.1055 / \mathrm{s}-0039-1695664$ ISSN 2277-954X.
(C)2019 Neurological Surgeons'

Society of India
License terms

() (1) $\odot \circledast$ 
artery that gives rise to a radiculomeningeal branch that supplies the dura mater and nerve root. Some of these radicular arteries will also supply the spinal cord as radiculomedullary or radiculopial arteries. A normal spinal cord has single anterior and paired posterior spinal arteries that receive their reinforcements from radicular branches of various segmental arteries. The venous drainage of the spinal cord is through intrinsic radially arranged veins that connect to pial venous network on the surface of spinal cord. The extrinsic venous drainage is by pial venous network and anterior and posterior spinal veins. The superficial veins surrounding the spinal cord drain into the radiculomedullary veins. These radiculomedullary veins accompany the nerve root exiting from the respective neural foramina and the dural sheath at this site. In addition, in about $40 \%$ individuals there are bridging veins that exit from the dura at sites other than nerve root sleeve. ${ }^{1,2}$ In case of spinal dAVF, the fistula lies inside the dura close to the intervertebral foramen, where one or more radiculomeningeal arteries have a fistulous communication with the radiculomeningeal vein. The consequent decrease in the AV pressure gradient leads to engorgement of the perimedullary venous plexuses, the extrinsic and intrinsic veins, and is thus responsible for a venous congestion resulting in myelopathy.

\section{Material and Methods}

Three cases of spinal dAVF were diagnosed over a period of 6 months. The patients were further evaluated with selective angiography to confirm the diagnosis, localize the pathology, and delineate the anatomy of fistula. Two cases had significant neurologic deficit, and the fistula was deemed to be the cause. The third case was incidentally detected and was not intervened. Neurosurgeons and interventional radiologists jointly discussed all three cases. The two operated cases were found to be unsuitable for intervention by neuroradiologists and were worked up for early surgical excision. The third case is under periodic follow-up.

\section{Results}

\section{Case 1}

A 65-year-old woman became symptomatic with paresthesia/tingling and numbness of the left (Lt) lower limb. Over the next 1 month, she developed similar symptoms in the right (Rt) lower limb and sought medical attention. The symptoms followed an indolent course for next 3 months wherein the patient started to have difficulty in prolonged or brisk walk. Climbing stairs became increasingly difficult, and she had tiredness of legs. Thereafter, she rapidly developed weakness in both lower limbs, and by the end of 4 months she had become wheelchair bound and required catheterization. So far, she had been treated for degenerative disc disease, and this rapid worsening prompted her referral to our center with a clinical diagnosis of multiple sclerosis. On examination, she had reduced sensation below D6 by around 20\% and could barely perceive touch and pinprick in sacral dermatomes. Tone was increased in lower limbs, and reflexes were brisk. Ankle clonus was present bilaterally. The patient had significant weakness of both lower limbs and could not stand even with support. An MRI of the spine revealed multiple dilated perimedullary veins along the dorsal spine. There was T2 hyperintensity extending from D5 to conus medullaris. There was postcontrast enhancement of the perimedullary veins. Angiography confirmed a spinal dAVF with feeder from radiculomeningeal artery along Lt D10 nerve root. The patient underwent a D10 laminectomy. A single arterial feeder was identified along the Lt D10 nerve root, and intradurally a single draining vein led to a conglomeration of perimedullary veins along dorsal aspect of the cord that appeared turgid and arterialized. The single draining vein was disconnected close to the dura. Although the conglomeration of arterialized veins appeared less turgid after disconnection, no significant change was felt intraoperatively. The patient subjectively claimed to be feeling better within 48 hours of surgery and was discharged on fifth postoperative day without any objective improvement in her neurologic status. She improved to some extent by second week post-surgery. Thereafter the improvement occurred rapidly and steadily. By around fourth week, she was walking with support, and by the end of 3 months, she had recovered complete power and had been voiding normally (-Table $\mathbf{1}$ ).

\section{Case 2}

A 69-year-old man had pin- and needle-like sensations in both the feet. These sensations were insidious in onset and persisted for a period of 3 to 4 months. By this time, the patient had developed reduced sensations in both the feet, and sensations of pins and needles had subsided completely. Subsequently, by around 6 months, he started to develop weakness of both the lower limbs. Initially the weakness was in the Rt ankle that progressed to involve the Rt knee and then hip over a period of 12 months. This was closely mirrored by weakness in the opposite limb. He was using a walking stick by the end of 12 months and could walk only by using a walker by 18 months. During this period, he was treated at various centers for multiple diagnoses such as degenerative disc disease, hyponatremia, and multiple sclerosis. By the time of presentation to our hospital, he had been symptomatic for a period of about 26 months and was wheelchair bound. He had been on steroids for 4 weeks with no improvement. Significant wasting of the thigh and calf muscles bilaterally was present. On examination he was found to have spastic quadriparesis with reduced sensation below D10 dermatome. A review of the previous MRI and a repeat MRI showed dilated perimedullary veins along the dorsal cord. A T2 hyperintensity was present from D9 to conus medullaris with contrast enhancement at D11-12 along with cord thickening at this level. A spinal angiography confirmed a spinal dAVF with feeder from radiculomeningeal artery along the Rt D9 nerve root. Laminectomy DV9 and partial laminectomy DV8 and DV10 was done. Intraoperatively single draining vein led to a plethora of arterialized veins on the dorsal surface of cord. The draining vein was ligated close to the dura. There were one dominant arterial feeder and an additional doubtful feeder along D10 nerve root. Both feeders were ligated. The patient had a slow but definite recovery. After 3 months, he still had residual 
Table 1 Clinical course of operated cases

\begin{tabular}{|c|c|c|c|c|c|c|c|c|c|c|c|c|}
\hline & \multicolumn{5}{|c|}{ Case 1} & \multicolumn{5}{|c|}{ Case 2} \\
\hline & & & Preoperative & $2 \mathrm{wk}$ & 4 wk & $8 \mathrm{wk}$ & $12 \mathrm{wk}$ & Preoperative & $2 \mathrm{wk}$ & $4 \mathrm{wk}$ & $8 w k$ & $12 \mathrm{wk}$ \\
\hline \multirow[t]{6}{*}{ Motor } & \multirow[t]{2}{*}{ Hip } & (Rt) & $2 / 5$ & $3 / 5$ & $4+/ 5$ & $5 / 5$ & $5 / 5$ & $2 / 5$ & $2 / 5$ & $2 / 5$ & $3 / 5$ & $4+/ 5$ \\
\hline & & (Lt) & $2 / 5$ & $3 / 5$ & $4+/ 5$ & $5 / 5$ & $5 / 5$ & $2 / 5$ & $2 / 5$ & $2 / 5$ & $2 / 5$ & $4+/ 5$ \\
\hline & \multirow[t]{2}{*}{ Knee } & (Rt) & $2 / 5$ & $2 / 5$ & $4 / 5$ & $5 / 5$ & $5 / 5$ & $1 / 5$ & $1 / 5$ & $1 / 5$ & $2 / 5$ & $4 / 5$ \\
\hline & & $(\mathrm{Lt})$ & $2 / 5$ & $2 / 5$ & $4 / 5$ & $5 / 5$ & $5 / 5$ & $1 / 5$ & $1 / 5$ & $1 / 5$ & $2 / 5$ & $4 / 5$ \\
\hline & \multirow[t]{2}{*}{ Ankle } & (Rt) & $2 / 5$ & $2 / 5$ & $2 / 5$ & $3 / 5$ & $5 / 5$ & $1 / 5$ & $1 / 5$ & $1 / 5$ & $2 / 5$ & $4 / 5$ \\
\hline & & $(\mathrm{Lt})$ & $2 / 5$ & $2 / 5$ & $2 / 5$ & $3 / 5$ & $5 / 5$ & $1 / 5$ & $1 / 5$ & $1 / 5$ & $2 / 5$ & $4 / 5$ \\
\hline $\begin{array}{l}\text { Bladder } \\
\text { control }\end{array}$ & & & On catheter & $\mathrm{SCIC}$ & $\mathrm{SCIC}$ & $\mathrm{SCIC}$ & Self-voiding & On catheter & \begin{tabular}{|l|} 
On \\
Catheter
\end{tabular} & $\mathrm{SCIC}$ & $\mathrm{SCIC}$ & Self-voiding \\
\hline $\begin{array}{l}\text { Sensa- } \\
\text { tions }\end{array}$ & & & $\begin{array}{l}50 \% \text { below } \\
\text { D6 }\end{array}$ & Better & Better & $\begin{array}{l}\text { Nearly } \\
\text { normal }\end{array}$ & Normal & $\begin{array}{l}\text { 20\% below } \\
\text { D10 }\end{array}$ & Better & $\begin{array}{l}50 \% \\
\text { below } \\
\text { D10 }\end{array}$ & \begin{tabular}{|l|}
$50 \%$ \\
below \\
normal
\end{tabular} & $\begin{array}{l}\text { Marginal- } \\
\text { ly below } \\
\text { normal }\end{array}$ \\
\hline
\end{tabular}

Abbreviation: SCIC, self-clean intermittent catheterization.

paraparesis and reduced sensations but was significantly better. He had started to void on his own (- Table 1).

\section{Case 3}

A 51-year-old woman reported to OPD with pain on lower back radiating to the Lt lower limb for about 3 months. The patient gave history of a fall from a height about 6 months previously. She had no neurologic deficits. She was evaluated with an MRI study and was found to have a fracture LV2 with anterior wedging and a syrinx from CV4 to DV4. Flow voids were noted at lower dorsal level suggestive of spinal dAVF. She further underwent an angiography, and presence of dAVF along the Lt D7 nerve root was confirmed. She was counseled in detail about the entity and future course. As she had no symptoms from the dAVF yet, a decision in line with patient's desire was taken to keep her under close periodic follow-up for onset of any neurologic deficits and intervene only if she develops any symptoms.

\section{Discussion}

Spinal dAVF is the most common spinal vascular anomaly and constitutes about $70 \%$ of all vascular malformations of the spine. ${ }^{3-5}$ Its incidence is very low and is a rare entity in clinical practice, which is often misdiagnosed. ${ }^{6-8}$ Both the patients who underwent surgery had been treated at other centers for 4 and 26 months before being diagnosed. Both operated cases based on their clinical findings of mixed upper motor neuron (UMN) and lower motor neuron (LMN) features had been previously evaluated for multiple sclerosis. The second patient had even been administered a course of steroids without any benefit. This was despite the patient having undergone MRI study where the significance of perimedullary veins was not appreciated, thus leading to a delayed diagnosis and truncated recovery. The patient was transferred to our center because of the progressive course of the disease despite a perceived optimal treatment. Reevaluation of previous study and a repeat study revealed possibility of dAVF, which was confirmed with a selective angiography. The presence of fistula was picked up incidentally in the third case, which had so far not been symptomatic.
Two cases were in seventh decade and one was in sixth decade. One was male and two were females. Mean age of occurrence in the literature has been reported to be 55 to 60 years, and it is five times more common in men. More than $80 \%$ cases occur between T6 and L2 with sacral and high cervical incidence being less than 4 and $2 \%$, respectively. ${ }^{3}$ All these three patients had fistula located between T6 and L2 levels. MRI picked up prominent perimedullary flow voids in all the three patients (-Figs. 1-3). Cord edema was seen in two of the patients who underwent surgical ligation subsequently. All the three patients on spinal digital subtraction angiography had a single radiculomeningeal feeder with dilated, tortuous perimedullary veins. Postoperative MRI was done after 1 month in both operated cases. First patient revealed complete resolution of the cord edema and perimedullary flow voids ( - Fig. 1e) whereas the second patient revealed reduction in the cord edema and disappearance of the perimedullary flow voids ( $\mathbf{- F i g . 2 e}$ ).

Pathologically a spinal dAVF involves an abnormal communication between a radiculomeningeal branch of the

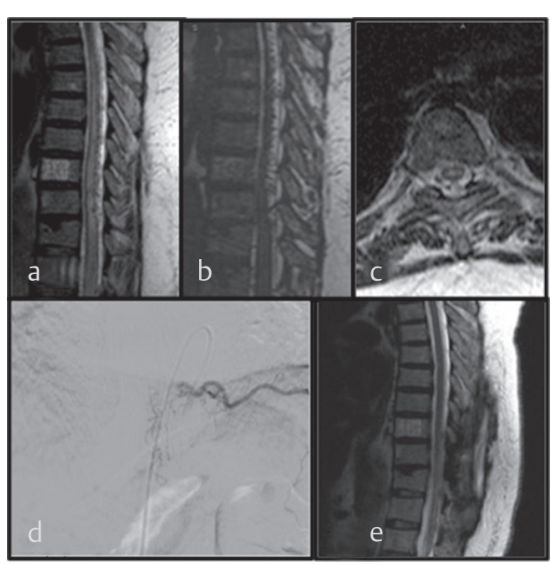

Fig. 1 (a) T2-weighted sagittal image showing edema in the dorsal cord. (b) CISS sagittal image showing perimedullary flow voids. (c) T2-weighted axial image showing dorsal cord edema. (d) DSA showing the spinal dAVF with feeder from (Lt) D10 radicular artery. (e) Postsagittal T2-weighted MRI showing complete resolution of the cord edema and perimedullary flow voids. 


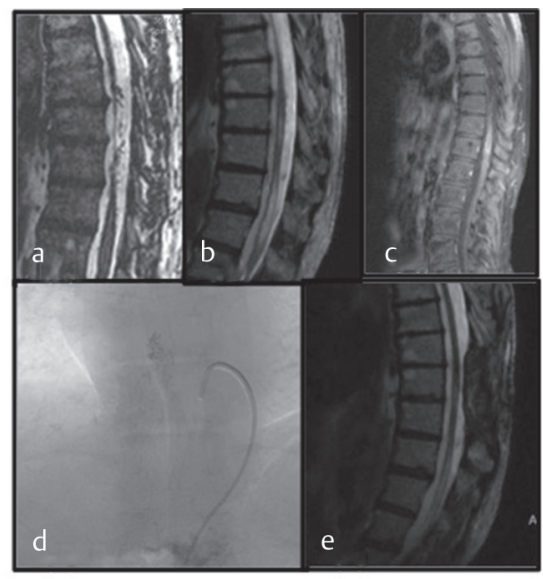

Fig. 2 (a) Sagittal CISS sequence showing prominent perimedullary veins. (b) T2-weighted sagittal sequence showing edema of the dorsal cord. (c) Postcontrast image showing enhancement of the edematous cord with perimedullary veins. (d) DSA showing spinal dAVF with feeder from (Rt) D9 radicular artery and dilated perimedullary veins. (e) Postoperative MRI sagittal T2-weighted sequence showing reduction in the perimedullary flow voids.

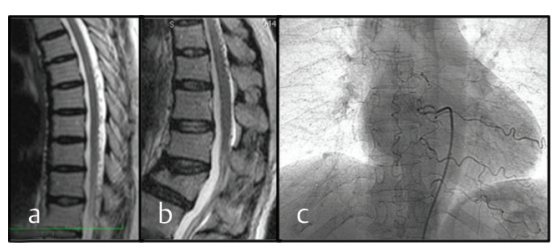

Fig. 3 (a) T2-weighted sequence of upper dorsal spine showing prominent perimedullary flow voids. (b) T2-weighted sequence of lower dorsal and upper lumbar spine showing continuation inferiorly of prominent perimedullary flow voids. Incidentally detected old fracture LV2. (c) DSA showing dAVF at D7 vertebral level.

segmental artery with a draining vein and has been discussed under anatomy. The exact cause of this fistula is not known, but it is presumed to be acquired. The excessive flow of arterial blood directly into draining veins causes high-pressure situation in a low-pressure system, leading to congestive changes in the respective draining area of the spinal cord.

The symptomatology is nonspecific with little to make a confident clinical diagnosis. Early symptoms include easy fatigability with nonspecific low backache that might aggravate after exertion because of rise in arterial pressure leading to increased pressure and flow through the fistula. Radicular pain may or may not occur. Sensory symptoms such as paresthesias/tingling/numbness, patchy or diffuse sensory loss and motor symptoms such as weakness of lower limbs, and difficulty in climbing stairs and/or walking have been reported. Involvement of bowel and bladder in the form of incontinence or retention of urine generally occurs later in the course of disease. $^{3}$ The disease runs a slow indolent course with progressive worsening. However, sudden onset, rapid worsening, and occasional remissions may also occur. ${ }^{9}$ Among our cases, one case had a slow progression of the disease, taking almost 2 years to develop significant deficits. First case, however, had a very rapid progression from onset of first symptom to becoming wheelchair bound. In both these cases, patients had sought medical attention in the early presentation of the disease. However, it was only when they became wheelchair bound that they got referred to tertiary care center leading to diagnosis of the disease. The initial symptomatology is so vague and inconspicuous that a rare entity such as spinal $\mathrm{dAVF}$ is not even considered in the differential diagnosis.

As the disease progresses slowly, by the time diagnosis is made, the patient has advanced deficits. Motor weakness along with bowel and bladder deficits might already be present before the diagnosis is made or even suspected. Foix and Alajouanine ${ }^{10,11}$ in their original description have described presence of both UMN and LMN lesions simultaneously in these patients.

Diagnosis is invariably made after MRI, which identifies the pathologic changes of dilated and congested venous channels with cord edema and/or myelopathy. Perimedullary veins appear as dilated serpentine flow voids predominantly located dorsal to the cord on T2-weighted sequences and seen as flow voids. These vessels can be further confirmed by using heavy T2-weighted sequences such as CISS (constructive interference in steady state) or FIESTA (fast imaging employing steady state acquisition). Cord edema is identified as hyperintensity seen on T2-weighted sequences extending over multiple segments with or without a hypointense rim around it because of deoxygenated blood present in the surrounding capillaries. The location of cord edema, however, is not suggestive of the location of fistula that may be located distant from the site of cord edema. ${ }^{12}$ Following contrast administration, enhancement will be seen in the perimedullary veins and sometimes in the cord due to breakdown in the blood-spinal cord barrier.

Superselective angiography remains the gold standard to delineate the anatomy of fistula and differentiate these from other AV malformations. However, this entails injecting contrast in each segmental artery to look for the fistula. Herein lies the significance of magnetic resonance angiography (MRA) and computed tomographic angiography (CTA) to suggest the possible location of fistula. ${ }^{13-17} \mathrm{CTA}$, however, has the disadvantage of radiation exposure, and hence, although it is informative, it has limited utility. A spinal dAVF on angiography appears as a slow-flow lesion with an arterial feeder from radiculomeningeal artery, bunch of feeding vessels from a lesion located invariably in the dura and converging into a draining vein. Dilated perimedullary veins due to back pressure may be seen either ascending or descending.

The treatment of spinal dAVF aims at its exclusion from the vascular system either by surgical excision or embolization. Surgical excision involves ligation of arterial feeder and the draining vein. The feeder artery has to be ligated closest to the dura as numerous collaterals may be present, and if it is ligated farther from the dura, it might give only transient relief until the collaterals become more active and nullify the benefits of ligation of the feeder. Similarly, the vein has to be ligated closest to the dura where the fistula zone lies. Embolization of this fistula involves superselective cannulation of the feeder, and nidus has to be passed before liquid embolic agent can be placed at the proximal part of the draining vein. Endovascular therapy is reported to have a success rate varying from 25 to $75 \%{ }^{9,18}$ Surgical excision of fistula has been found to have a success rate of $98 \% .^{19}$ 


\section{Conclusion}

Spinal dAVF is more common than what is perceived. Numerous patients remain undiagnosed till advanced stages of clinical deterioration. Spinal dAVFs are surgically curable and show remarkable recovery. An early diagnosis can be made with increased awareness of this entity and early intervention.

\section{Conflicts of Interest}

None.

\section{Contributions}

1. Dr Manish Sharma: Conceptualization, data collection, manuscript writing.

2. Dr Ankit Mathur: Radiologic evaluation, manuscript writing.

\section{References}

1 Kiyosue H, Matsumaru Y, Niimi Y, et al; JSNET Spinal AV Shunts Study Group. Angiographic and clinical characteristics of thoracolumbar spinal epidural and dural arteriovenous fistulas. Stroke 2017;48(12):3215-3222

2 Miyasaka K, Asano T, Ushikoshi S, Hida K, Koyanagi I. Vascular anatomy of the spinal cord and classification of spinal arteriovenous malformations. Interv Neuroradiol 2000;6(Suppl 1):195-198

3 Krings T, Geibprasert S. Spinal dural arteriovenous fistulas. AJNR Am J Neuroradiol 2009;30(4):639-648

4 Kendall BE, Logue V. Spinal epidural angiomatous malformations draining into intrathecal veins. Neuroradiology 1977;13(4):181-189

5 Merland JJ, Riche MC, Chiras J. Intraspinal extramedullary arteriovenous fistulae draining into the medullary veins. J Neuroradiol 1980;7(4):271-320

6 Grandin C, Duprez T, Stroobandt G, Laterre EC, Mathurin P. Spinal dural arterio-venous fistula: an underdiagnosed disease? Acta Neurol Belg 1997;97(1):17-21

7 Jellema K, Tijssen CC, Sluzewski M, van Asbeck FW, Koudstaal PJ, van Gijn J. Spinal dural arteriovenous fistulas-an underdiagnosed disease. A review of patients admitted to the spinal unit of a rehabilitation center. J Neurol 2006;253(2):159-162

8 Jellema K, Tijssen CC, van Gijn J. Spinal dural arteriovenous fistulas: a congestive myelopathy that initially mimics a peripheral nerve disorder. Brain 2006;129(Pt 12):3150-3164

9 Van Dijk JM, TerBrugge KG, Willinsky RA, Farb RI, Wallace MC. Multidisciplinary management of spinal dural arteriovenous fistulas: clinical presentation and long-term follow-up in 49 patients. Stroke 2002;33(6):1578-1583

10 Foix $\mathrm{CH}$, Alajouanine $\mathrm{T}$. La myelite necrotique subaigue. Rev Neurol (Paris)1926;46:1-42

11 Schrader V, Koenig E, Thron A, Dichgans J. Neurophysiological characteristics of spinal arteriovenous malformations. Electromyogr. Clin Neurophysiol 1989;29(3):169-177

12 Bostroem A, Thron A, Hans FJ, Krings T. Spinal vascular malformations-typical and atypical findings. Zentralbl Neurochir 2007;68(4):205-213

13 Farb RI, Kim JK, Willinsky RA, et al. Spinal dural arteriovenous fistula localization with a technique of first-pass gadolinium-enhanced MR angiography: initial experience. Radiology 2002;222(3):843-850

14 Mull M, Nijenhuis RJ, Backes WH, Krings T, Wilmink JT, Thron A. Value and limitations of contrast-enhanced MR angiography in spinal arteriovenous malformations and dural arteriovenous fistulas. AJNR Am J Neuroradiol 2007;28(7):1249-1258

15 Bowen BC, Pattany PM. Contrast-enhanced MR angiography of spinal vessels. Magn Reson Imaging Clin N Am 2000;8(3):597-614

16 Bowen BC, Saraf-Lavi E, Pattany PM. MR angiography of the spine: update. Magn Reson Imaging Clin $\mathrm{N}$ Am 2003;11(4):559-584

17 Yamaguchi S, Eguchi K, Kiura Y, et al. Multi-detector-row CT angiography as a preoperative evaluation for spinal arteriovenous fistulae. Neurosurg Rev 2007;30(4):321-326, discussion 327

18 Niimi Y, Berenstein A, Setton A, Neophytides A. Embolization of spinal dural arteriovenous fistulae: results and follow-up. Neurosurgery 1997;40(4):675-682, discussion 682-683

19 Steinmetz MP, Chow MM, Krishnaney AA, et al. Outcome after the treatment of spinal dural arteriovenous fistulae: a contemporary single-institution series and meta-analysis. Neurosurgery 2004;55(1):77-87, discussion $87-88$ 